\title{
Physical adsorption at the nanoscale: Towards controllable scaling of the substrate-adsorbate van der Waals interaction
}

\author{
Alberto Ambrosetti, ${ }^{1}$ Pier Luigi Silvestrelli, ${ }^{1}$ and Alexandre Tkatchenko ${ }^{2}$ \\ ${ }^{1}$ Dipartimento di Fisica e Astronomia, Università degli Studi di Padova, via Marzolo 8, 35131, Padova, Italy \\ ${ }^{2}$ Physics and Materials Science Research Unit, University of Luxembourg, L-1511 Luxembourg \\ (Received 23 December 2016; revised manuscript received 14 March 2017; published 12 June 2017)
}

\begin{abstract}
The Lifshitz-Zaremba-Kohn (LZK) theory is commonly considered as the correct large-distance limit for the van der Waals (vdW) interaction of adsorbates (atoms, molecules, or nanoparticles) with solid substrates. In the standard approximate form, implicitly based on local dielectric functions, the LZK approach predicts universal power laws for $\mathrm{vdW}$ interactions depending only on the dimensionality of the interacting objects. However, recent experimental findings are challenging the universality of this theoretical approach at finite distances of relevance for nanoscale assembly. Here, we present a combined analytical and numerical many-body study demonstrating that physical adsorption can be significantly enhanced at the nanoscale. Regardless of the band gap or the nature of the adsorbate specie, we find deviations from conventional LZK power laws that extend to separation distances of up to 10-20 nm. Comparison with recent experimental observations of ultra-long-ranged vdW interactions in the delamination of graphene from a silicon substrate reveals qualitative agreement with the present theory. The sensitivity of vdW interactions to the substrate response and to the adsorbate characteristic excitation frequency also suggests that adsorption strength can be effectively tuned in experiments, paving the way to an improved control of physical adsorption at the nanoscale.
\end{abstract}

DOI: 10.1103/PhysRevB.95.235417

Noncovalent van der Waals (vdW) interactions constitute a universal cohesive force whose impact extends from the atomistic scale $[1,2]$ to a wealth of macroscopic phenomena observed on a daily basis [3,4]. With an influence ranging from protein-drug binding to the double helix in DNA [5], the peculiar pedal adhesion in the gecko [6,7], and even cohesion in regolith and rubble-pile asteroids [8,9], these nonbonded forces are quantum mechanical in origin and arise from electrodynamic interactions between the constantly fluctuating electron clouds that characterize molecules and materials [10]. While our understanding of vdW interactions is rather complete at the smallest atomistic and the largest macroscopic scales, these pervasive forces exhibit a range of surprising and poorly understood effects at the nanoscale [10-16].

This lack of understanding is best exemplified by recent puzzling experimental observations, which include (i) ultralong-range $\mathrm{vdW}$ interactions extending up to tens of nanometers into heterogeneous $\mathrm{Si} / \mathrm{SiO}_{2}$ dielectric interfaces $[17,18]$, and influencing the delamination of extended graphene layers from silicon substrate [19]; (ii) complete screening of the vdW interaction between an atomic force microscope (AFM) tip and a $\mathrm{SiO}_{2}$ surface by the presence of a single layer of graphene adsorbed on the surface [20]; (iii) superlinear sticking power laws for the physical adsorption of metallic clusters on carbon nanotubes with increasing surface area [21]; and (iv) nonlinear increases in the vdW attraction between homologous molecules and an $\mathrm{Au}(111)$ surface as a function of the molecular size [22]. Recently, theoretical evidence was found for exceptionally long-ranged $\mathrm{vdW}$ attraction between coupled low-dimensional nanomaterials with metallic character [11] or small band gap [10,14]. Observed major deviations from conventional pairwise predictions [10] stem from nonlocal dipolar fluctuations induced by the low dimensionality of the structures [23].
While analysis of these striking phenomena focused on coupled one-dimensional (1D) and two-dimensional (2D) nanomaterials, the broader and technologically relevant problem of physical adsorption of atoms, molecules, or nanoparticles on low-dimensional structures is not yet fully understood. This lack of comprehension is mostly related to the intrinsically local charge fluctuations of small adsorbates and the non-negligible highest occupied molecular orbital and lowest unoccupied molecular orbital gaps, which may suggest weak coupling to the soft delocalized polarization modes of the substrate. However, transient electronic excitations in low-dimensional substrates could cause unexpectedly strong electrodynamic fields, whose effects are yet to be assessed.

Both energetics and dynamical properties of physically adsorbed moieties can largely depend on the precise vdW scaling. Experimental implications of possible unexpected trends in nanoscale physical adsorption can thus range from catalysis and wetting to film deposition or self-assembly. Stateof-the-art single-molecule AFM experiments are now also able to measure power-law exponents governing the adsorption energy of large molecules on solid substrates to a precision of \pm 0.2 [22]. Such experimental progress provides a substantial challenge for the theoretical understanding of $\mathrm{vdW}$ interactions and precise modeling of their effects at the nanoscale. To achieve both goals, here we utilize a combined analytic and numerical many-body model of physical adsorption to systematically study the interaction of adsorbates with a range of both metallic and finite-gap low-dimensional 1D and 2D substrates. Even for the smallest atomic adsorbates, we find that the strongly nonlocal response of these substrates, which stems from coherent wavelike electronic fluctuations, causes qualitative deviations from conventional vdW energy predictions. In fact, the vdW adsorption energy can exhibit a peculiar slow decay over length scales extending from $\sim 5 \AA$ to well above $10 \mathrm{~nm}$. Interestingly, the interaction 
energy decay can be further regulated by a suitable choice of the substrate response and of the adsorbate moiety, thus opening a plethora of pathways towards detailed and selective experimental control of $\mathrm{vdW}$ forces at the nanoscale.

So far, the theoretical modeling of the complex many-body $\mathrm{vdW}$ interactions arising on extended substrates has mostly relied on the Lifshitz-Zaremba-Kohn (LZK) theory [24-26]. In principle the LZK approach provides an exact theoretical framework, where explicit dependence on the interacting substrate response function $\chi_{S}$ ensures complete inclusion of many-body screening effects. Due to the intrinsic complexity of $\chi_{\mathrm{S}}$, however, the interacting susceptibility is normally approximated in LZK calculations by an implicitly local form. Essentially, by approximating the substrate response in terms of the average dielectric function (computed at wave vector $\mathbf{q}=0$ ) the complexity of the problem can be strongly reduced, and simple power-law expressions can be derived for the $\mathrm{vdW}$ interaction energy $\Delta E_{\mathrm{vdW}}$. This is exemplified, for instance, by the well-known expression $\Delta E_{\mathrm{vdW}} \sim C_{3} / D^{3}$, derived for small molecular fragments at large distance $D$ from a semiinfinite substrate, and extended (with different power-law dependence on $D$ ), to treat also lower-dimensional substrates [27,28]. Within the LZK approach the overall Hamaker constants $\left(C_{3}\right.$ in the above expression) are renormalized with respect to standard pairwise vdW approximations [29-33], due to the effective inclusion of screening effects in the extended substrate. However, the vdW interaction power laws predicted in the local LZK limit exactly coincide with those of additive pairwise $\mathrm{vdW}$ approaches [for a general definition of the interaction power law, see Eq. (SM2) in the Supplemental Material [34], which also includes Refs. [35,36]]. While this approach is generally correct for bulklike substrates, here we will analyze in detail the implications of the locality approximation, evidencing major shortcomings in the rapidly emerging context of low-dimensional substrates. By explicitly accounting for nonlocal electron charge fluctuation we will thus provide a correct application of the LZK theory to substrates with arbitrary dimensionality and response properties.

In order to introduce the essential physical concepts, we begin our analysis by considering a single adsorbate $A$ (for instance an atom, a molecule, or a nanoparticle) interacting with a $1 D$ metallic wire $W$ at a separation $D$. Atomic units ( $e=m=4 \pi \epsilon_{0}=\hbar=1$ ) are adopted hereafter to simplify the notation. The wire density-density response $\chi_{\mathrm{W}}^{\mathrm{RPA}}$ can be computed starting from the 1D free electron gas bare susceptibility $[37,38] \chi_{\mathrm{W}}^{0}(q, \omega)=N_{0} q^{2} / \omega^{2}$, where $N_{0}$ is the number of electrons per unit length, and the intrawire Coulomb interaction [11] $v_{\mathrm{W}}(q)=-2 e^{2} \ln (q b)$ ( $b$ being the effective wire thickness, in the limit $b q \ll 1)$. The $\mathrm{vdW}$ interaction energy is thus evaluated by coupling the polarizability $\alpha_{A}(i \omega)$ of the adsorbate to the RPA interacting substrate response through the wire-adsorbate interaction [11] $2 K_{0}(q D)$ (a modified Bessel function of the second kind; see Ref. [25]), as

$$
\Delta E_{\mathrm{vdW}}=\int_{0}^{\infty} d \omega \int d q \frac{q^{2} I(q D)}{\pi^{2}} \chi_{\mathrm{W}}^{\mathrm{RPA}}(q, i \omega) \alpha_{\mathrm{A}}(i \omega) .
$$

Here $I(q D)=\left[K_{0}(q D)^{2}+K_{0}^{\prime}(q D)^{2}\right]$, and momentum integration is restricted to the first Brillouin zone. For $D$ larger than the adsorbate characteristic dimension, the dipole approximation can be adopted for the response of the adsorbate $A$. We also map now the adsorbate polarizability onto the response of a single quantum harmonic oscillator making use of the single Lorentzian expression $\alpha_{\mathrm{A}}(i \omega)=\alpha_{\mathrm{A}}^{0} /\left[1+\left(\omega^{2} / \omega_{\mathrm{A}}^{0}\right)^{2}\right]$ (being $\alpha_{\mathrm{A}}^{0}$ the static polarizability and $\omega_{\mathrm{A}}^{0}$ the characteristic oscillator frequency). This procedure corresponds to introducing a single effective excitation mode for the adsorbate, and has been widely applied in literature $[27,39,40]$. Alternative treatments based on multiple excitation modes, however, are equally possible within this framework, and can be reformulated in terms of linear combinations of the single mode contributions considered hereafter. After analytical frequency integration, the following expression is obtained:

$$
\Delta E_{\mathrm{vdW}}=-\int d q \frac{q^{2} I(q D)}{2 \pi} \frac{\alpha_{\mathrm{A}}^{0} \omega_{\mathrm{A}}^{0} q N_{0}}{L(q)\left(\omega_{\mathrm{A}}^{0}+q L(q)\right)},
$$

where $L(q)=\sqrt{2|\ln (q b)| N_{0}}$. We note that, at variance with conventional LZK theory, the explicit $q$ dependence of the substrate response function is preserved in this derivation, thus accounting for the actual nonlocality of the charge fluctuation modes.

To study the scaling of $\Delta E_{\mathrm{vdW}}$ with respect to the adsorption distance, we first observe that the rapidly decaying interaction factor $I(q D)$ introduces an effective integration cutoff at $q \sim 1 / D$. By performing the variable substitution $q^{\prime}=q D$, it becomes thus evident that the power-law scaling of $E_{\mathrm{vdW}}$ is determined by the $q$ dependence of the integrand [10,11], and it specifically varies depending on the relative magnitude of the terms at the denominator [namely, $\omega_{\mathrm{A}}^{0}$ and $q L(q)$ ]. In particular, we can distinguish two separate regimes: (i) For $\omega_{\mathrm{A}}^{0} \gg L(1 / D) / D$ the integrand is roughly proportional to $I(q D) q^{3} / L(q)$ over the whole integration domain. Integration over $q$ thus leads to $E_{\mathrm{vdW}} \sim D^{-4}$ up to logarithmic corrections. (ii) If the opposite case holds [i.e., $\omega_{\mathrm{A}}^{0} \ll L(q) q$ over most of the integration domain], then the integrand becomes roughly proportional to $I(q D) q^{2} / L^{2}(q)$, leading to power-law scalings that are intermediate between $\sim D^{-3}$ and $\sim D^{-4}$. According to the above analysis, by increasing $D$ regime (i) is eventually approached, and the transition between regimes (ii) and (i) is influenced by the adsorbate characteristic frequency $\omega_{\mathrm{A}}^{0}$ : in fact, for small values of $\omega_{\mathrm{A}}^{0}$ the $\sim D^{-4}$ scaling is approached at larger adsorption distances (see Fig. 1). At high $\omega_{\mathrm{A}}^{0}$, instead, regime (i) is soon approached, but the power law can show a slight initial growth due to the logarithmic corrections, remaining, however, close to the $\sim D^{-4}$ asymptote. Based on the above analysis, we note that the vdW interaction energy between substrate and adsorbate exhibits evident qualitative deviations from conventional pairwise predictions $\left(D^{-5}\right.$ in 1D), implying an ultraslow decay of the interaction with respect to the adsorption distance. The existence of separate scaling ranges, moreover, suggests that the interaction details may be experimentally tuned by an appropriate choice of the adsorbate species: physically speaking, adsorbates with different $\omega_{\mathrm{A}}^{0}$ will be sensitive to different frequency ranges, and, correspondingly, to distinct characteristic modes of the substrate. As from Fig. 1(b), we also observe that a decrease of $\omega_{\mathrm{A}}^{0}$ leads at short distances to weaker vdW attraction, compensated at large $D$ by the slower decay. Clearly [see 

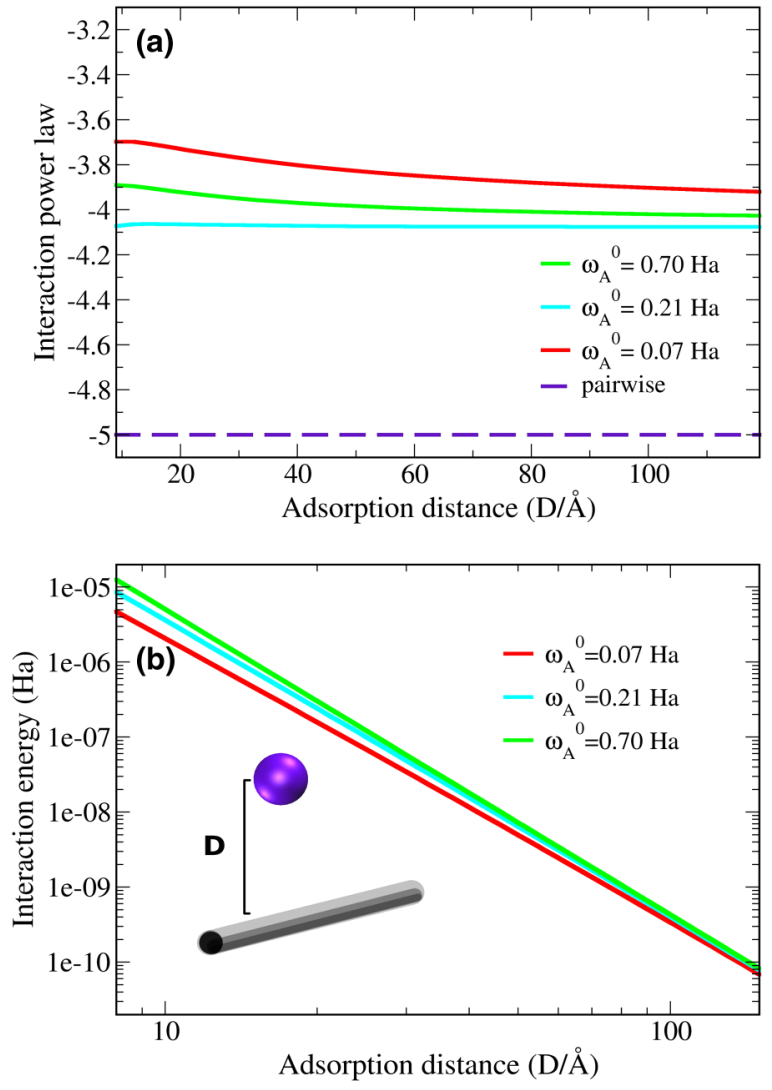

FIG. 1. Power law decay (a) of the vdW interaction, and vdW interaction energy in logarithmic scale (b) for a single adsorbate on an infinite metallic wire $\left(N_{0}=1\right.$ bohrs $\left.^{-1}\right)$. Adsorbates with different characteristic frequency $\omega_{\mathrm{A}}^{0}$ are considered in order to visualize the interdependence between the adsorbate dynamical polarizability and the interaction power law. The adsorbate static polarizability is kept fixed to the reference value of an isolated $\mathrm{Ar}$ atom (11.1 $\mathrm{bohrs}^{3}$ ). Comparison with the standard pairwise power law $\left(D^{-5}\right)$ indicates more evident deviations from the pairwise behavior in adsorbates with lower characteristic frequency. We also note from Eq. (2) that the static polarizability $\alpha_{\mathrm{A}}^{0}$ can be factorized, hence only providing a constant overall rescaling of the vdW interaction.

Eq. (1)] the vdW energy is also proportional to $\alpha_{\mathrm{A}}^{0}$. An increase in the static polarizability combined with a decrease in the characteristic frequency could thus be exploited in order to achieve consistent vdW enhancement at all ranges.

Similar conclusions can be drawn by considering the tightbinding response function for 1D metallic chains proposed by Misquitta et al. [14]. In that case, however, the nonlocality of $\chi_{\mathrm{w}}$ entirely derives from the collective character of the quasiparticle eigenstates, and does not stem from the selfconsistent RPA treatment of the Coulomb coupling.

While the above results are specifically derived for a metallic 1D substrate, we now extend our treatment to finite-gap structures. Due to electronic charge localization one can describe in this case the response of an $N$-atom system in terms of $N$ interacting atomic polarizabilities. These can be mapped onto a set of coupled atom-centered quantum harmonic oscillators, as outlined by the many-body dispersion (MBD) framework [10,41], by introducing the coupled dipolar
Hamiltonian $[42,43]$ :

$$
\begin{aligned}
H_{\mathrm{MBD}}= & -\sum_{p=1}^{N} \frac{\nabla_{\boldsymbol{\mu}_{p}}^{2}}{2}+\sum_{p=1}^{N} \frac{\omega_{p}^{2} \boldsymbol{\mu}_{p}^{2}}{2} \\
& +\sum_{p \neq q}^{N} \omega_{p} \omega_{q} \sqrt{\alpha_{p}^{0} \alpha_{q}^{0}} \boldsymbol{\mu}_{p} T_{p q} \boldsymbol{\mu}_{q} .
\end{aligned}
$$

The $p$ th atom is characterized by the static polarizability $\alpha_{p}^{0}$ and the characteristic frequency $\omega_{p}$, and $\boldsymbol{\mu}_{p}$ describes the mass-weighted charge displacement from the ionic position $\mathbf{R}_{p}$ [43]. The interaction tensor $T$ introduces a dipolar coupling between different oscillators, and is defined as $T_{p q}=$ $\nabla_{\mathbf{R}_{p}} \nabla_{\mathbf{R}_{q}} v\left(R_{p q}\right)$, where $v\left(R_{p q}\right)$ is the Coulomb interaction between atoms $p$ and $q$, damped at short range due to Gaussian charge overlap [44]. Given the quadratic dependence on $\boldsymbol{\mu}_{p}, H_{\mathrm{MBD}}$ can be exactly diagonalized, leading to a set of $3 N$ interacting frequencies $\bar{\omega}_{p}$, from which the vdW energy can be promptly computed as $E_{\mathrm{vdW}, \mathrm{MBD}}=$ $\left(3 \sum_{p=1}^{N} \omega_{p}-\sum_{p=1}^{3 N} \bar{\omega}_{p}\right) / 2$. The resulting dispersion energy is mathematically equivalent to the random phase approximation (RPA) [44] long-range correlation energy arising between dipolar oscillators.

In order to investigate adsorption on 1D nonconducting systems, we consider a carbynelike wire, consisting of a linear chain of $\mathrm{C}$ atoms with equal nearest-neighbor distances $d_{\mathrm{C}-\mathrm{C}}$. Different values of $d_{\mathrm{C}-\mathrm{C}}$ are then analyzed, in order to assess the role of the chain response. In Fig. 2, we observe that, also in finite-gap systems, sizable deviations from the the conventional $D^{-5}$ scaling are possible even beyond $\sim 10 \mathrm{~nm}$. In analogy with the case of two parallel 1D chains [10], the power law initially increases reaching a plateau, and subsequently decreases, gradually tending to the pairwise limit. Again, the effect is enhanced by low adsorbate characteristic frequencies. Moreover, power-law deviations become evidently more pronounced at smaller $d_{\mathrm{C}-\mathrm{C}}$ values, while the vdW energy scaling rapidly approaches $D^{-5}$ beyond $d_{\mathrm{C}-\mathrm{C}}=2.0 \AA$. The ultraslow decay of vdW interactions is thus closely related to the nonlocality of the dipolar response of the chain. In fact, as observed in Ref. [10], highly collective dipolefluctuation modes can emerge in low-dimensional structures. Such modes correspond to the dipole waves sustained by the system, and characterize the degree of nonlocality of the response function. Within the MBD approach these collective wavelike modes are directly obtained as the eigenmodes of the Hamiltonian (3), and depending on the dispersion of the corresponding eigenenergies $[\bar{\omega}(q)]$, different power-law scalings of the vdW energy can be found.

This concept can be formalized by expressing the vdW adsorption energy $\Delta E_{\mathrm{vdW}, \mathrm{MBD}}$ in integral form. We thus take the continuum limit (valid at large $D$ ), and consider only the longitudinal dipole fluctuation modes occurring in the chain. Transverse modes, in fact, provide smaller contributions to $\Delta E_{\mathrm{vdW}, \mathrm{MBD}}$ at large $D$ and will be neglected for simplicity. Making explicit use of the $f$-sum rule [45], we can express the polarizability of the collective mode corresponding to wave vector $q$ as $\bar{\alpha}(q, i \omega)=\bar{\alpha}^{0}(q) /\left[1+\omega^{2} / \bar{\omega}^{2}(q)\right]$, where the static polarizability is written in terms of the C static polarizability $\alpha_{\mathrm{C}}^{0}$ and the characteristic frequency $\omega_{\mathrm{C}}^{0}$ as $\bar{\alpha}^{0}(q)=\alpha_{\mathrm{C}}^{0}\left[\omega_{\mathrm{C}}^{0} / \bar{\omega}(q)\right]^{2}$. 

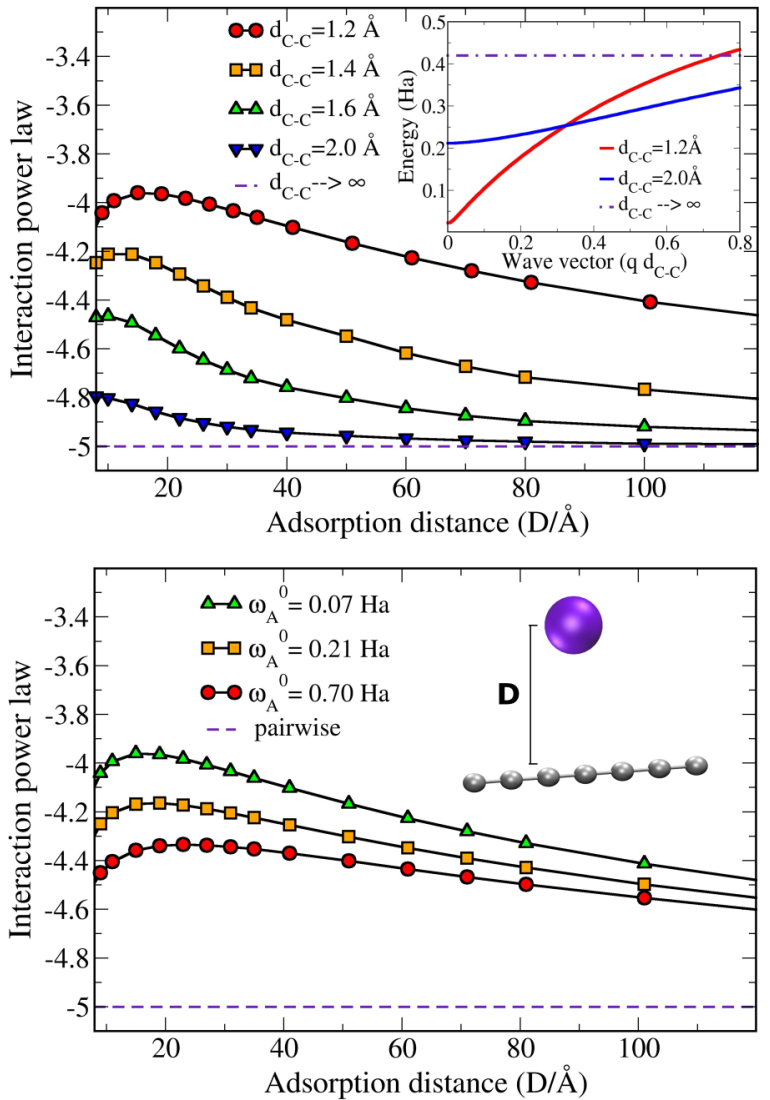

FIG. 2. MBD power-law decay of the vdW interaction between single adsorbate (with characteristic frequency $\omega_{\mathrm{A}}^{0}=0.07 \mathrm{Ha}$ ) and a periodic 1D atomic chain. Upper panel: chains with different interatomic distance $d_{\mathrm{C}-\mathrm{C}}$ are considered. The larger deviations from the pairwise limit $D^{-4}$ observed at small $d_{\mathrm{C}-\mathrm{C}}$ find a correspondence in the steep mode dispersion and small energy gap reported in the inset. In fact, the steep dispersion is indicative of a strongly nonlocal dipolar response in the chain. Lower panel: dependence of the interaction power law on the adsorbate frequency $\left(\omega_{\mathrm{A}}^{0}\right)$. In analogy to the metallic case, adsorbates with lower $\omega_{\mathrm{A}}^{0}$ exhibit more evident deviations from the pairwise $D^{-5}$ limit.

By extending Eq. (2) to the present model, we can thus express the interaction energy as

$$
\Delta E_{\mathrm{vdW}, \mathrm{MBD}}=-\int d q \frac{I(q D)}{2 \pi} \frac{\alpha_{\mathrm{A}}^{0} \alpha_{\mathrm{C}}^{0} \omega_{\mathrm{A}}^{0}\left(\omega_{\mathrm{C}}^{0} q^{2}\right)^{2}}{\bar{\omega}(q)\left(\bar{\omega}(q)+\omega_{\mathrm{A}}^{0}\right)} .
$$

In analogy with Eq. (2), the mode dispersion $\bar{\omega}(q)$ entering at the denominator ultimately determines the power-law scaling of $\Delta E_{\mathrm{vdW}, \mathrm{MBD}}$. For instance, at large $d_{\mathrm{C}-\mathrm{C}}$ the atoms in the chain become weakly interacting, leading to flat energy dispersion $[\bar{\omega}(q) \sim$ const]. In the presence of energetic degeneracy, localized dipole fluctuations can thus occur in the system and the pairwise approximation becomes valid. At realistic interatomic distances, instead, the intrachain interaction acts by lifting the modes degeneracy, leading to nontrivial mode dispersion (see Fig. 2). In particular, one observes that $\bar{\omega}(q)$ can assume very small values for $q \rightarrow 0$ [10], showing then a steep increase at growing $q$. The steep dispersion of the charge fluctuation modes is a clear signature of response nonlocality (see Supplemental Material). We stress, though, that due to
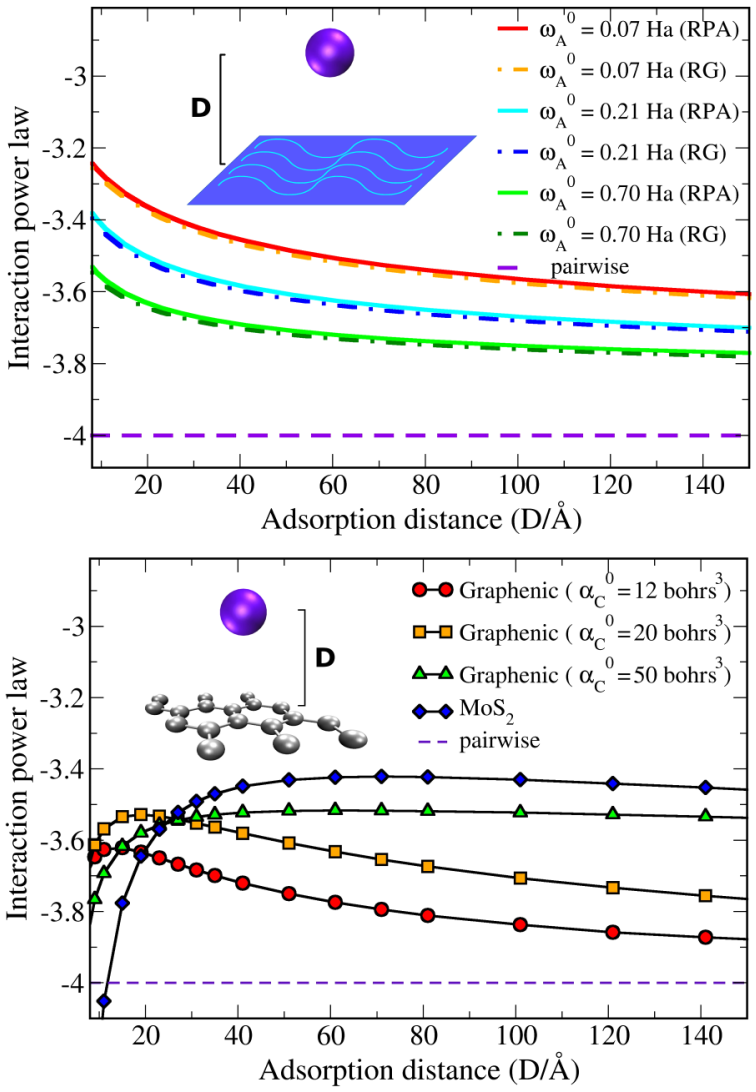

FIG. 3. Power-law decay of the vdW interaction between single atoms and 2D materials. Upper panel: $\Delta E_{\mathrm{vdW}}$ computed at different adsorbate characteristic frequencies, adopting analytical RPA and RG response functions for graphene. Lower panel: MBD results for $2 \mathrm{D}$ materials characterized by graphenic structure and variable atomic polarizability $\left(\omega_{\mathrm{A}}^{0}=0.07 \mathrm{Ha}\right)$. The pairwise asymptotic limit $D^{-4}$ is reported for comparison.

the intrinsic localization of the single quantum harmonic oscillators (justified by the electron charge localization), $\bar{\omega}(0)$ is always nonzero. This property determines a qualitative asymptotic difference with respect to the metallic case, implying that the $D^{-5}$ power law is recovered as the asymptotic limit.

To extend our treatment beyond 1D substrates, we now consider the adsorption on a 2D graphene substrate. Given the complexity of the full electronic structure, we make use of approximate response functions, based on the low-energy excitations of the $\pi$ electrons. Although this approximation neglects polarization components orthogonal to the plane, nonetheless it permits one to unravel the effects of the band structure near the Dirac cone, that govern the nontrivial electronic properties of graphene. Besides the conventional RPA $[11,46]$ response function, a more accurate approximation is also considered, derived including vertex corrections through a renormalization group (RG) approach $[12,47]$. Details on the response functions, and on the computation of $\Delta E_{\mathrm{vdW}}$ using this approach are reported in the Supplemental Material. From Fig. 3, a clear analogy emerges between adsorption on 1D systems and graphene. Sizable power-law deviations from the pairwise limit extend beyond $100 \AA$, and are again enhanced in the presence of adsorbates with low characteristic 
frequency. Moreover, the semiquantitative agreement existing between power laws derived within RPA and RG suggests that ring diagrams can already account for relevant response delocalization, hence providing further support to the present MBD results.

In order to unravel how vdW interactions depend on the substrate details, we apply the MBD method to single-layer $\mathrm{MoS}_{2}$ and finite-gap graphenic materials, setting the atomic polarizability $\alpha_{\mathrm{C}}^{0}$ to different values. Interestingly, by inspection of Fig. 3, we find qualitative agreement between atomistic MBD calculations and the previous semianalytical approach based on the response of $\pi$ electrons only. In addition, we observe that the more polarizable substrates (such as graphenic materials with higher $\alpha_{\mathrm{C}}^{0}$ or $\mathrm{MoS}_{2}$ ) [48] are characterized by a slower decay of the adsorption energy with respect to $D$. The analogies existing between higher $\alpha_{\mathrm{C}}^{0}$ and smaller $d_{\mathrm{C}-\mathrm{C}}$ (see Fig. 2), can be understood considering that many-body effects in MBD are effectively controlled by the dimensionless quantity $\alpha_{\mathrm{C}}^{0} / d_{\mathrm{C}-\mathrm{C}}^{3}$. An inverse proportionality thus exists between power-law variations induced by changes in the two quantities. The important deviations found for $\mathrm{MoS}_{2}$ also suggest that other quasimetallic or finite-gap low-dimensional materials, such as transition metal dichalcogenides, silicene, or phosphorene should exhibit analogous trends in physical adsorption processes.

Going from monolayer graphenic structures to multilayer graphene (see Supplemental Material), pairwise power laws are gradually approached at short $D$, suggesting that the conventional vdW asymptotic decay should be typically recovered in bulk systems. At the same time, however, deviations from pairwise power laws become longer-lasting with respect to $D$ when increasing the number of layers: at large $D$ thin multilayered structures effectively behave as a single layer with enhanced polarizability-to-surface ratio, thus inducing longer-ranged vdW interactions which require an appropriate description beyond the local LZK limit.

We finally note that extremely long-ranged interlayer forces have been observed in a very recent experiment [19] conducted by separating graphene from the native oxide layer on a Si(111) substrate by lateral wedge insertion and crack opening. While $\mathrm{vdW}$ interactions are expected to contribute up to the $\sim 10 \mathrm{~nm}$ scale, the experimentally estimated delamination resistance per unit area only converged to a constant value at $\sim 1 \mu \mathrm{m}$ crack openings. To interpret this puzzling result we considered cracks with longitudinal extent $a$ and quadratic increase of the graphene-Si(111) separation $h(x)$ with respect to the crack coordinate $x \in[0, a]$ (see Supplemetal Material). By assuming $\mathrm{a} \sim h(x)^{-2.5}$ power-law scaling of the interaction (a variation of 0.5 from the pairwise power law is compatible with our findings), we found that the dispersion energy cost for crack formation (per unit area) approaches its converged value within $2 \%$ only beyond $h(a) \sim 0.5 \mu \mathrm{m}$. Considering instead cracks with constant opening $h$ and a $h^{-3}$ interaction scaling, the dispersion energy cost is converged within $2 \%$ already at $h \sim 2 \mathrm{~nm}$. Our simple analysis can thus qualitatively capture the observed ultra-long-ranged sticking effect. Moreover, the combined many-body polarization enhancement in graphene [49], and the complex strain effects occurring in the system upon mechanical deformation could further extend the effective range of the effective interlayer interaction.

In conclusion, we evidenced highly nontrivial power-law scalings of the vdW interaction arising between atoms or small molecules and both metallic and finite-gap low-dimensional substrates. These power laws substantially deviate from standard pairwise predictions, and result in ultra-long-ranged dispersion forces. This effect arises due to marked nonlocalities of the substrate response, and could only be captured by accounting for the detailed momentum dependence of the susceptibility within a full many-body approach. The nontrivial dispersion enhancements predicted for atomic adsorbates demonstrate that any type of systemfrom the atomistic scale up to the nanoscale-can undergo ultra-long-ranged vdW forces in the presence of polarizable low-dimensional substrates. The sensitivity of the vdW energy scaling to the adsorbate characteristic frequency and substrate response properties paves the way to a detailed and selective control of molecule-substrate interactions. These results open new perspectives for challenging experimental manipulations of adsorption and nanoassembly phenomena. Possible implications may also extend to the broad context of low-dimensional biological systems, including phospholipid aggregates and bilayers [50], or extended polypeptide chains [51].

A.A. acknowledges useful and insightful discussion with Flavio Toigo.
[1] A. J. Stone, The Theory of Intermolecular Forces, 2nd ed. (Oxford University Press, Oxford, UK, 2013).

[2] I. G. Kaplan, Intermolecular Interactions (Wiley-Blackwell, Hoboken, 2006).

[3] D. Langbein, Theory of Van der Waals Attraction, Springer Tracts in Modern Physics Vol. 72 (Springer, Berlin, 1974).

[4] V. A. Parsegian, Van der Waals Forces: A Handbook for Biologists, Chemists, Engineers and Physicists (Cambridge University Press, New York, 2005).

[5] R. A. DiStasio, Jr., O. A. von Lilienfeld, and A. Tkatchenko, Proc. Natl. Acad. Sci. USA 109, 14791 (2012).

[6] K. Autumn, Y. A. Liang, S. T. Hsieh, W. Zesch, W. P. Chan, T. W. Kenny, R. Fearing, and R. J. Full, Nature (London) 405, 681 (2000).
[7] K. Autumn, M. Sitti, Y. A. Liang, A. M. Peattie, W. R. Hansen, S. Sponberg, T. W. Kenny, R. Fearing, and R. J. Full, Proc. Natl. Acad. Sci. USA 99, 12252 (2002).

[8] B. Rozitis, E. MacLennan, and J. P. Emery, Nature (London) 512, 174 (2014).

[9] D. J. Scheeres, C. M. Hartzell, P. Sánchez, and M. Swift, Icarus 210, 968 (2010).

[10] A. Ambrosetti, N. Ferri, R. A. DiStasio, Jr., and A. Tkatchenko, Science 351, 1171 (2016).

[11] J. F. Dobson, A. White, and A. Rubio, Phys. Rev. Lett. 96, 073201 (2006).

[12] J. F. Dobson, T. Gould, and G. Vignale, Phys. Rev. X 4, 021040 (2014).

[13] D. Chang, R. Cooper, J. Drummond, and A. C. Young, Phys. Lett. A 37, 311 (1971). 
[14] A. J. Misquitta, J. Spencer, A. J. Stone, and A. Alavi, Phys. Rev. B 82, 075312 (2010).

[15] A. J. Misquitta, R. Maezono, N. D. Drummond, A. J. Stone, and R. J. Needs, Phys. Rev. B 89, 045140 (2014).

[16] C. A. Silvera Batista, R. G. Larson, and N. A. Kotov, Science 350, 1242477 (2015).

[17] P. Loskill, H. Hähl, T. Faidt, S. Grandthyll, F. Müller, and K. Jacobs, Adv. Colloid Interface Sci. 179, 107 (2012).

[18] P. Loskill, J. Puthoff, M. Wilkinson, K. Mecke, K. Jacobs, and K. Autumn, J. R. Soc. Interface 10, 20120587 (2013).

[19] S. R. Na, J. W. Suk, R. S. Ruoff, R. Huang, and K. M. Liechti, ACS Nano 8, 11234 (2014).

[20] S. Tsoi, P. Dev, A. L. Friedman, R. Stine, J. T. Robinson, T. L. Reinecke, and P. E. Sheehan, ACS Nano 8, 12410 (2014).

[21] G. Rance, D. Marsh, S. Bourne, T. Reade, and A. Khlobystov, ACS Nano 4, 4920 (2010).

[22] C. Wagner, N. Fournier, V. G. Ruiz, C. Li, K. Müllen, M. Rohlfing, A. Tkatchenko, R. Temirov, and F. S. Tautz, Nat. Commun. 5, 5568 (2014).

[23] D. Y. Qiu, F. H. da Jornada, and S. G. Louie, Phys. Rev. B 93, 235435 (2016).

[24] E. M. Lifshitz, Sov. Phys. JETP 2, 73 (1956).

[25] E. Zaremba and W. Kohn, Phys. Rev. B 13, 2270 (1976).

[26] G. L. Klimchitskaya, U. Mohideen, and V. M. Mostepanenko, Rev. Mod. Phys. 81, 1827 (2009).

[27] E. Cheng and M. W. Cole, Phys. Rev. B 38, 987 (1988).

[28] M. Bordag, B. Geyer, G. L. Klimchitskaya, and V. M. Mostepanenko, Phys. Rev. B 74, 205431 (2006).

[29] S. Grimme, J. Antony, S. Ehrlich, and H. Krieg, J. Chem. Phys. 132, 154104 (2010)

[30] A. Tkatchenko and M. Scheffler, Phys. Rev. Lett. 102, 073005 (2009).

[31] M. Dion, H. Rydberg, E. Schroder, D. C. Langreth, and B. I. Lundqvist, Phys. Rev. Lett. 92, 246401 (2004).

[32] P. L. Silvestrelli, Phys. Rev. Lett. 100, 053002 (2008).
[33] E. R. Johnson and A. D. Becke, J. Chem. Phys. 123, 024101 (2005).

[34] See Supplemental Material at http://link.aps.org/supplemental/ 10.1103/PhysRevB.95.235417 for a detailed description of computational details, including relevant parameters, power-laws definition, the analytical model response function of graphene, and the theoretical approach to graphene delamination.

[35] D. D. L. Chung, J. Mater. Sci. 37, 1475 (2002).

[36] J. F. Dobson and A. Rubio, arXiv:cond-mat/0502422.

[37] P. Longe and S. M. Bose, Phys. Rev. B 48, 18239 (1993).

[38] G. Giuliani and G. Vignale, Quantum Theory of the Electron Liquid (Cambridge University Press, Cambridge UK, 2008).

[39] M. J. T. Oliveira, S. Botti, and M. A. L. Marques, Phys. Chem. Chem. Phys. 13, 15055 (2011).

[40] L. W. Bruch, M. W. Cole, and E. Zaremba, Physical Adsorption: Forces and Phenomena (Clarendon, Oxford, UK, 1997).

[41] A. Tkatchenko, R. A. DiStasio, Jr., R. Car, and M. Scheffler, Phys. Rev. Lett. 108, 236402 (2012).

[42] W. L. Bade, J. Chem. Phys. 27, 1280 (1957).

[43] A. G. Donchev, J. Chem. Phys. 125, 074713 (2006).

[44] A. Tkatchenko, A. Ambrosetti, and R. A. DiStasio, Jr., J. Chem. Phys. 138, 074106 (2013).

[45] D. Pines, Elementary Excitations in Solids: Lectures on Phonons, Electrons, and Plasmons (Westview Press, Boulder, 1999).

[46] J. González, F. Guinea, and M. A. H. Vozmediano, Nucl. Phys. B 424, 595 (1994).

[47] I. Sodemann and M. M. Fogler, Phys. Rev. B 86, 115408 (2012).

[48] According to the Tkatchenko-Scheffler parametrization, $\mathrm{MoS}_{2}$ is characterized by an average polarizability per unit area which is about four times larger than in graphene.

[49] V. V. Gobre and A. Tkatchenko, Nat. Commun. 4, 2341 (2013).

[50] L. J. Lis, M. McAlister, N. Fuller, R. P. Rand, and V. A. Parsegian, Biophys. J. 37, 667 (1982).

[51] F. Schubert et al., Phys. Chem. Chem. Phys. 17, 7373 (2015). 